BMJ Open

Diabetes

Research

\& Care

\section{The effect of macrobiotic Ma-Pi 2 diet on systemic inflammation in patients with type 2 diabetes: a post hoc analysis of the MADIAB trial}

To cite: Soare A, Del Toro $R$, Roncella $\mathrm{E}$, et al. The effect of macrobiotic Ma-Pi 2 diet on systemic inflammation in patients with type 2 diabetes: a post hoc analysis of the MADIAB trial. BMJ Open Diabetes Research and Care 2015:3:e000079. doi:10.1136/bmjdrc-2014000079

- Additional material is available. To view please visit the journal online (http://dx. doi.org/10.1136/bmjdrc2014-000079)

Received 23 December 2014 Revised 8 February 2015 Accepted 11 March 2015

\section{(a) CrossMark}

For numbered affiliations see end of article.

Correspondence to Professor Paolo Pozzilli; p.pozzilli@unicampus.it

\section{ABSTRACT}

Introduction: Current guidelines for the management of type 2 diabetes (T2D) emphasize diet as essential therapy. However, the effect of diet on systemic inflammation remains unclear. We investigated the effects of consuming a macrobiotic Ma-Pi 2 diet versus a standard recommended diet (control diet) on markers of inflammation in patients with T2D. Methods: This was a post hoc analysis of the MADIAB trial, a 21-day randomized controlled trial conducted in 51 patients (25 males and 26 females) with T2D. Patients were randomized 1:1 to the Ma-Pi 2 macrobiotic diet or a control diet based on dietary guidelines for T2D. Biological antioxidant potential of plasma and circulating levels of high-sensitivity $C$ reactive protein, interleukin- 6 , tumor necrosis factor- $\alpha$, and insulin-like growth factor-1 were assessed.

Results: After 21 days on the Ma-Pi 2 or control diet, markers of inflammation were reduced in both groups. The antioxidant potential of plasma improved significantly in the Ma-Pi group. A significant reduction in insulin growth factor- 1 was observed in the Ma-Pi group versus control group $(p<0.001)$.

Conclusions: Findings of this post hoc analysis demonstrated that the Ma-Pi 2 diet is a safe dietary strategy to reduce levels of the markers of insulin resistance and inflammation, compared with baseline values, in the short term. Furthermore, the Ma-Pi 2 diet was superior to the control diet in reducing insulin growth factor- 1 and may be beneficial for patients with T2D.

Trial registration number: Current Controlled Trials ISRCTN10467793.

Type 2 diabetes (T2D) is one of the major public health challenges of the 21st century. Diet and lifestyle directly influence the health status of a population, and rates of T2D continue to increase with the global trend toward a greater prevalence of obesity and reduced physical activity. ${ }^{1}$ Major risk factors for T2D have also been found to induce local or systemic low-grade chronic

\section{Key messages}

Results of this post hoc analysis of the MADIAB Trial, a 21-day randomized controlled trial in patients with type 2 diabetes, showed that the macrobiotic Ma-Pi 2 diet is a safe dietary treatment to reduce levels of the inflammatory markers in the short term.

- The Ma-Pi 2 diet was superior to the standard recommended diet for type 2 diabetes in reducing insulin resistance, insulin growth factor-1.

- Further studies are required to elucidate whether the short term changes in inflammatory markers obtained with the Ma-Pi 2 diet are maintained over a longer time.

inflammation. Systemic inflammation is independently associated with insulin resistance, as supported by studies conducted in adults ${ }^{2}$ and adolescents. ${ }^{3} 4$

Chronic low-grade inflammation has been hypothesized as an underlying pathophysiological mechanism linking obesity to T2D. ${ }^{3}$ Tumor necrosis factor (TNF) $\alpha$ is a potent cytokine produced by adipose tissue that induces the production of interleukin- 6 (IL-6). ${ }^{5}$ Plasma levels of TNF are persistently elevated in patients with obesity or T2D, and the role of TNF in promoting insulin resistance is mediated by an increase in adipocyte lipolysis. ${ }^{6}$ Because TNF is the first step in the inflammatory cascade, it is not surprising that plasma levels of IL-6 are high in patients with obesity or T2D. Similarly, IL-6 concentrations also correlate with insulin resistance in humans. ${ }^{7}$ One of the most important effects of IL-6 is that it controls hepatic $\mathrm{C}$ reactive protein (CRP) production. CRP is a key inflammatory marker in humans, and is positively associated with degree of obesity, and fasting glucose and insulin levels. ${ }^{8}$ 
Oxidative stress is caused by an imbalance between reactive oxygen species (ROS) and antioxidant molecules. Both endogenous and exogenous compounds play a role as antioxidants in living organisms to keep reactive species and free radicals down to physiological levels. Oxidative stress and inflammation together play a crucial part in the development of many chronic diseases such as obesity, T2D, and cardiovascular and neurodegenerative diseases. Furthermore, imbalances in ROS, free radicals, and antioxidants have been highlighted as major mechanisms underlying obesity-related comorbidities. ${ }^{9} 10$ Altering nutrition may be an important means of reducing oxidative stress, since composition of the diet has been shown to strongly affect the body's antioxidant capacity. ${ }^{11}$ Blood antioxidant potential (BAP) provides a reliable measurement of the antioxidant barrier power to oxidation. ${ }^{12}$

Under physiological conditions during the feedingfasting cycle, a non-specific and intermittently low-grade inflammatory response occurs in the metabolic tissues (adipose, muscular, and hepatic), which determines a transient increase in serum inflammatory cytokines like CRP, IL-6 and TNF- $\alpha$. This increase reaches its peak during the postprandial phase and then decreases gradually during the following $2 \mathrm{~h}^{13}$

The inflammatory response is amplified by high consumption of red meat, processed meat, refined grains, simple carbohydrates, high-fat dairy products, and low intake of fiber and antioxidants. Obesity and diabetes can develop due to the resulting metabolic overload, creating a block between immune and metabolic functions (insulin/insulin growth factor-1). ${ }^{14}$

Dietary intervention, which is probably the key determinant of the balance that influences the overall inflammatory hallmark, represents a valid method of assessing the role of diet in the prevention of chronic inflammation. ${ }^{15}$ The assessment of whole dietary patterns has become instrumental in nutritional epidemiology. Taking into account that nutrients or foods are rarely eaten in isolation, dietary patterns must consider synergistic or antagonistic biochemical interactions among nutrients as well as different food sources of the same nutrient. ${ }^{16} 17$

During the past two decades, evidence from prospective observational studies and clinical trials has converged to support the importance of individual nutrients, foods, and dietary patterns in the prevention and management of inflammation. A systematic review investigating dietary patterns and changes in biomarkers of inflammation showed some evidence to support the hypothesis that so called 'Western-like' or meat-based patterns are positively associated with low-grade inflammation, and that vegetable-based and fruit-based patterns appeared to be inversely related to inflammatory biomarkers ${ }^{18}$; this was particularly well supported by intervention studies investigating the Mediterranean diet. ${ }^{19}$

A cross-sectional study of 902 women with T2D from the Nurses' Health Study reported that consumption of a diet high in dietary fiber was associated with higher plasma levels of adiponectin than diets low in fiber; adiponectin is a molecule with anti-inflammatory properties that protects against diabetes and atherosclerosis. ${ }^{20}$ In two additional cross-sectional studies, plasma levels of CRP were negatively related to total fiber intake both in healthy adult participants, ${ }^{21}$ and in patients with diabetes, hypertension, or obesity (from the National Health and Nutrition Examination Survey). ${ }^{22}$ These associations persisted when variables were adjusted for other confounding dietary and non-dietary factors.

The Ma-Pi 2 macrobiotic diet is a low-fat, high-fiber, high-complex carbohydrate, mainly vegetarian diet conceived by Mario Pianesi. As recently demonstrated by our group in the first randomized trial that assessed the effects of the Ma-Pi 2 diet versus the standard nutritional recommendations for T2D (MADIAB trial), patients consuming the Ma-Pi 2 diet experienced statistically significant improvements in terms of reduced fasting blood glucose (FBG), postprandial blood glucose (PPBG), glycated hemoglobin (HbAlc), insulin resistance, and greater weight loss, compared with patients receiving the control diet, despite both diets having the same energy content. ${ }^{23}$ However, the potential of this diet for reducing markers of inflammation requires additional investigation.

The present study is a post hoc analysis of the MADIAB trial, ${ }^{23}$ and aims to evaluate the effect of the two dietary strategies on inflammatory markers, insulin-like growth factor-1 (IGF-1), and BAP among patients with T2D.

\section{RESEARCH DESIGN AND METHODS \\ Participants}

Full details of the MADIAB trial have been reported previously. ${ }^{23}$ In summary, the MADIAB trial ${ }^{23}$ was a 21-day randomized controlled open-label study in which 56 participants were randomized in a 1:1 ratio to receive the Ma-Pi 2 macrobiotic diet or a control diet based on dietary guidelines for T2D. ${ }^{24}$ The inclusion criteria were overweight or obese (body mass index (BMI) $27-45 \mathrm{~kg} / \mathrm{m}^{2}$ ) males and females, aged 40-75 years, with a diagnosis of T2D at least 1 year prior to the start of the trial, and management with dietary intervention or oral hypoglycemic drugs, or both, for 6 months prior to study entry.

The primary outcomes of this study were the percentage change in FBG and PPBG levels from baseline to the 21st day of treatment in the Ma-Pi 2 group compared with the control group. Secondary outcomes included percentage change from baseline in plasma concentrations of HbA1c, total cholesterol, low-density lipoprotein (LDL) cholesterol, high-density lipoprotein (HDL) cholesterol, LDL/HDL ratio, percentage change of insulin resistance, body weight, BMI, waist and hip circumference, and number of patients who achieved target values of FBG $\leq 110 \mathrm{mg} / \mathrm{dL}$ and PPBG $\leq 140 \mathrm{mg} / \mathrm{dL}$. $^{23}$

Throughout the trial, participants stayed at two different hotels according to the type of diet they were randomized to; the hotels were localized in the same geographic area. 
Patients were recruited from the Endocrinology and Diabetes Department at University Campus Bio-Medico in Rome.

The study was approved by the Ethics Committee of the University Campus Bio-Medico of Rome (11/13 PAR ComEt CBM) and registered at http://www.ISRCTN.org (ISRCTN10467793). All of the participants provided written informed consent for participation in agreement with the Declaration of Helsinki. This work was performed in accordance with the CONSORT 2010 guidelines, ${ }^{25}$ and follow-up visits were conducted fortnightly.

Anthropometric measurements were assessed and blood samples for biochemical parameters, inflammation markers and antioxidant potential of plasma taken at the beginning and end of the 3-week study period. Blood samples were obtained for all participants in both groups. Food record data were also available for all participants in the study. During the intervention period, participants were asked not to alter their exercise habits, and physical activity was assessed daily using a pedometer (Tri-axial activity monitor XL-18/XL-18 CN-AND A\&D Medical, California, USA).

\section{Diets}

The experimental diets contained different levels of protein $(12 \%$ total caloric value (TCV) in the Ma-Pi 2 diet vs $18 \% \mathrm{TCV}$ in the control diet) and dietary fiber $(29 \mathrm{~g} / 1000 \mathrm{kcal}$ in the Ma-Pi 2 diet vs $21 \mathrm{~g} / 1000 \mathrm{kcal}$ in the control diet). The rest of the macronutrients were distributed as follows: $73 \%$ TCV from carbohydrates plus $15 \%$ TCV from fats in the Ma-Pi 2 diet and 49\% TCV from carbohydrates plus $32 \%$ TCV from fats in the control diet.

The Ma-Pi 2 diet consisted of whole grains, vegetables, and legumes. Beicha tea (roasted green tea) represented the main source of liquids. The control diet was adapted to the Mediterranean culinary style. The diets were isocaloric but differed in nutrient composition, as described above. For both groups, energy intake was restricted by limiting calories to 1900 and $1700 \mathrm{kcal} /$ day for males and females, respectively. Further information on the diets and how compliance was maintained is available in the primary MADIAB manuscript ${ }^{23}$ and online supplementary file 1. Dietary adherence was defined as absence of any transgression from the assigned diet. Participants who attended the meal sessions for fewer than 20 out of the 21 days were considered non-adherent.

Diet compositions were determined with the use of MètaDieta software, using the Italian Food Composition Tables edited by the National Institute for Food and Nutrition Research (INRAN), which has been described previously. $^{26}$

\section{Anthropometric and biochemical assessments}

Anthropometric measurements, including body weight and waist and hip circumference, were assessed at baseline and at the end of the intervention using a digital scale accurate to $0.1 \mathrm{~kg}$ and in accordance with validated protocols. $^{27}$ BMI was calculated as the body weight divided by the squared height $\left(\mathrm{kg} / \mathrm{m}^{2}\right)$.

Insulin sensitivity in the fasting state was assessed with homeostasis model assessment (HOMA) and calculated with the following formula: fasting plasma glucose $(\mathrm{mmol} / \mathrm{L}) \times$ fasting serum insulin $(\mu \mathrm{U} / \mathrm{mL})$ divided by 25, as described by Matthews et $a l^{28}$ high HOMA scores denote low insulin sensitivity (insulin resistance). Assays for fasting serum insulin and plasma glucose levels were performed by the central laboratory (University Campus Bio-Medico, Rome). Plasma insulin levels were assayed by radioimmunoassay (Ares, Serono, Italy).

Plasma concentrations of high-sensitivity CRP (Dimension Vista System), IL-6 (R\&D Systems), TNF- $\alpha$ (R\&D Systems), and IGF-1 (Cusabio System) were measured with specific ELISA kits from specified suppliers (shown in brackets), with the use of an autoanalyzer system (Triturus, Grifols SA, Rome, Italy) in accordance with the manufacturers' instructions.

Serum samples for cytokine and CRP levels were stored at $-80^{\circ} \mathrm{C}$ until assayed. Serum concentrations of IL- 6 and TNF- $\alpha$ were determined in duplicate using a highly sensitive, quantitative sandwich enzyme assay (Quantikine HS, R\&D Systems, Minneapolis, Minnesota, USA). The lower limit of detection was $0.7 \mathrm{pg} / \mathrm{mL}$ for both.

The BAP was measured using a commercial kit (Diacron, Grosseto, Italy). The BAP test provides an estimate of the global antioxidant capacity of blood plasma, measured as its reducing potential against ferric ions. Briefly, a $10 \mu \mathrm{L}$ serum sample is added to a solution of ferric chloride and thiocyanate derivate, and the intensity of any resulting absorbance change is proportional to the ability of plasma to reduce ferric ions. ${ }^{12}$ Photometric reading was employed to assess the absorbance change at $593 \mathrm{~nm}$. The intensity of the absorbance change is proportional to the ability of plasma to reduce ferric ions. ${ }^{12}$ The assay was performed on multiple well plates and absorbance readings taken on a number of 51 (Tecan).

\section{Statistical analyses}

A non-parametric approach was chosen for the statistical analysis since outcome variables were found to be of non-normal distribution (using the Shapiro test). Quantitative variables were summarized using percentiles (median and IQR). Comparisons between treatment groups at baseline were performed using the Wilcoxon rank-sum test and 95\% CIs for median values. Absolute and percentage frequencies were used for qualitative variables and the Fisher exact test was applied for group comparisons.

A bivariate analysis was performed to compare changes in inflammatory markers between the Ma-Pi 2 diet group and the control group, considering the absolute differences between values at basal (T0) and after 21 days of diet (T21) for TNF- $\alpha$ and IL-6, and the percent changes between T0 and T21 for CRP, BAP, and IGF-1. When the $95 \%$ CIs of the median difference (absolute or percent) did not include zero, the change 
was considered significantly different from zero. Results were graphically represented by means of box-plots.

A linear quantile regression analysis was performed to estimate the effect of the diet (Ma-Pi 2 vs control as explanatory variable) on the median absolute or percent change occurring in each measured variable (dependent variables), between $\mathrm{T} 0$ and T21. Each model was adjusted for those variables that could potentially affect the percent changes in the dependent variables, that is, gender, age, BMI at baseline, and physical activity of participants during 21 days of treatment (measured as the median number of kilometers walked per day).

The results of linear quantile regression analysis were expressed as point and interval estimates of regression coefficients; when the coefficient was positive, the measured variable reduction was related to Ma-Pi 2 diet effect and when the coefficient was negative the reduction was related to the control diet. When the 95\% CIs did not include zero, regression coefficients were considered statistically significant.

Power analysis was performed for the main outcomes in the MADIAB trial, ${ }^{23}$ but not for the additional outcomes reported in this post hoc analysis.

Owing to the nature of the trial, investigators at the hotels could not be blinded to the treatment allocation of participants. However, investigators analyzing blood samples and those collecting follow-up data were blinded from treatments.

All statistical analyses were performed using $\mathrm{R}$ statistical package (Foundation for Statistical Computing, Vienna, Austria) and statistical significance was assessed at a level of probability of 0.05 .

\section{RESULTS}

A total of 200 patients were screened and 56 were considered eligible for recruitment. The 56 eligible patients were randomly assigned to the Ma-Pi 2 diet group $(n=28)$ and the control group $(n=28)$. Reasons for exclusion were failure to meet inclusion criteria $(n=90)$, inability to attend residential schedule $(n=45)$, and failure to keep interview appointment $(n=9)$. The CONSORT diagram reflecting flow of study participants through the study is shown in figure 1.

Both groups experienced dropouts after randomization but before receiving any intervention, three patients in the Ma-Pi 2 diet group and two in the control group. Reasons for discontinuation included patient reluctance to change diet (1 patient in the Ma-Pi 2 group) and personal problems that prevented patients remaining in the assigned hotel for the study duration (2 patients in the Ma-Pi 2 group and 2 patients in the control group). Therefore, a total of 51 patients, 25 in the Ma-Pi 2 group and 26 in the control group, completed the trial and were included in the statistical analysis.

During the study, the daily average energy intake was 1803 (SD 95.2) kcal (11.8\% protein, $15.2 \%$ fat, and $73.0 \%$ carbohydrates, with $29 \mathrm{~g} / 1000 \mathrm{kcal}$ fiber) in the Ma-Pi 2 group, and 1798 (SD 106.3) kcal (18.4\% protein, $32.3 \%$ fat, and $49.3 \%$ carbohydrates, with $20.5 \mathrm{~g} / 1000 \mathrm{kcal}$ fiber) for the control group ( $\mathrm{p}=0.860$ ). At T21, participants in the Ma-Pi 2 group showed a significantly higher intake of carbohydrates and dietary fiber whereas a greater total protein intake was found in the control group.

The median body weight reduction from baseline was $5.5 \pm 1.0$ and $2.8 \pm 1.3 \mathrm{~kg}$ in the Ma-Pi 2 group and control group, respectively; weight loss was significantly higher in the Ma-Pi 2 group $(\mathrm{p}<0.0001)$. After 3 weeks of dietary intervention, participants in the Ma-Pi 2 group also demonstrated a significantly greater reduction in BMI, fasting, and postprandial glucose and insulin resistance compared with controls; data are presented and discussed in detail in the primary manuscript. ${ }^{23}$

\section{Figure 1 Consort diagram}

reflecting flow of study

participants through the study.

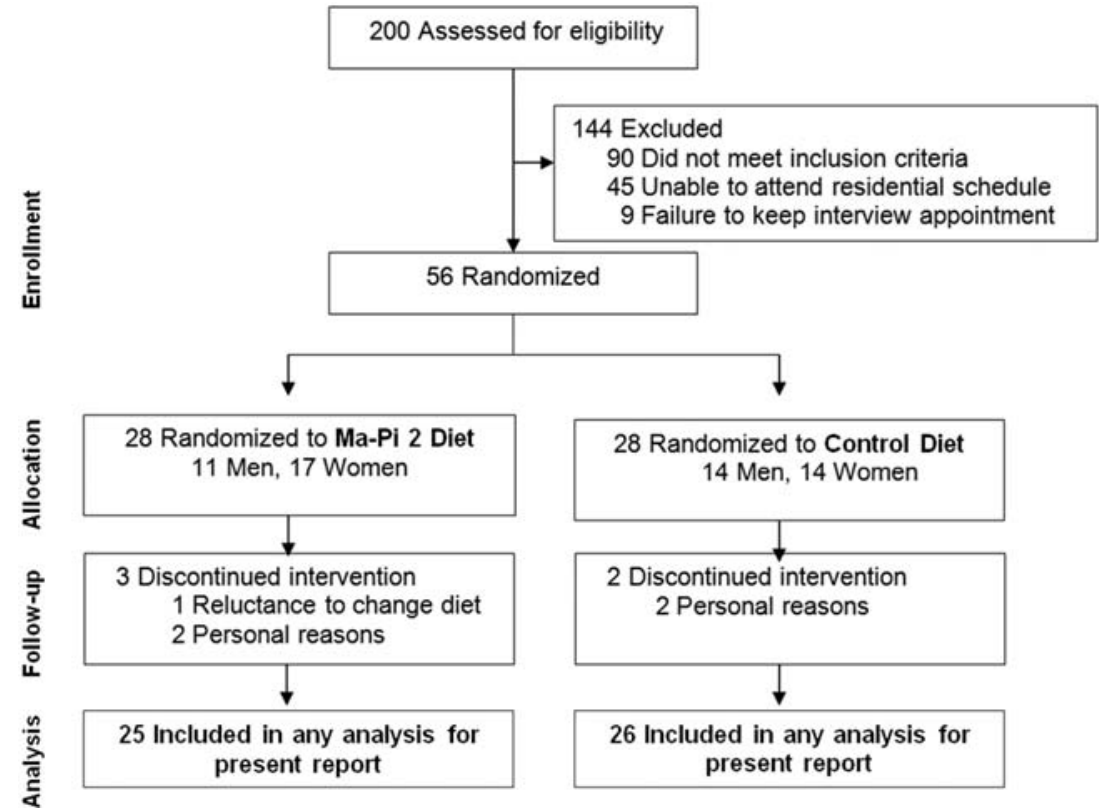


Table 1 Baseline characteristics of the participants according to study group

\begin{tabular}{lccc}
\hline Median $\left(\mathbf{1}^{\circ}-\mathbf{3}^{\circ}\right.$ quartile) & Ma-Pi $\mathbf{2}(\mathbf{n}=\mathbf{2 5})$ & Control $(\mathbf{n}=\mathbf{2 6})$ & $\mathbf{p ~ V a l u e ~}$ \\
\hline CRP $(\mathrm{mg} / \mathrm{L})$ & $3.2(1$ to 12$)$ & $2.5(1.4$ to 4.8$)$ & 0.769 \\
TNF- $\alpha(\mathrm{pg} / \mathrm{mL})$ & $23(0$ to 36$)$ & $25(11$ to 40$)$ & 0.296 \\
IL-6 $(\mathrm{pg} / \mathrm{mL})$ & $4.3(0$ to 6.4$)$ & $3.2(0$ to 5.5$)$ & 0.231 \\
BAP test $(\mathrm{mmol} / \mathrm{L})$ & $2109(1780$ to 2456$)$ & $1997(1721$ to 2275$)$ & 0.456 \\
IGF-1 $(\mathrm{ng} / \mathrm{mL})$ & $126(101.2$ to 192.7$)$ & $96.9(58.9$ to 162.8$)$ & 0.163
\end{tabular}

Wilcoxon rank-sum test.

Values are median $\left(1^{\circ}-3^{\circ}\right.$ quartile).

BAP test, biological antioxidant potential of plasma; CRP, C reactive protein; IGF-1, insulin-like growth factor-1; IL-6, interleukin-6; TNF- $\alpha$, tumor necrosis factor- $\alpha$.

Inflammatory biomarkers and the antioxidant potential of plasma (BAP) were assessed in the two groups at $\mathrm{T} 0$ and T21. No significant differences were found between the two groups in terms of TNF- $\alpha$, IL-6, CRP, BAP, and IGF-1 measured at baseline (table 1 and figure 2). At the end of the study, inflammation (measured by TNF- $\alpha$ and CRP) was significantly reduced in both groups. There were no statistical differences in levels of TNF- $\alpha$ or CRP between the two groups at T21. In addition, there was no significant change from baseline in IL-6 levels in either group, and there was no significant difference between groups at T21 ( $\mathrm{p}=0.888$; table 2). However, BAP improved significantly only in the Ma-Pi 2 group $(26.9 \%$ (95\% CI $9.1 \%$ to $44.7 \%)$ ). A significant median percent reduction of IGF-1 $(-12.6 \%$ (95\% CI $-24 \%$ to $-1.1 \%)$ ) was registered only in the Ma-Pi 2 group and IGF-1 levels were significantly improved in the Ma-Pi 2 group compared with the control group at T21 $(\mathrm{p}<0.001$; table 2$)$.
The results of linear quantile regression analysis (adjusted for age, gender, BMI at baseline, and physical activity) are reported in figure 3. A significantly higher percentage reduction of IGF-1 was observed in the Ma-Pi 2 group versus the control group, while higher absolute increases of IL-6 were associated with the control diet. No statistically significant differences were observed on the variation of TNF- $\alpha$, PCR, or BAP between groups.

Compliance was good in all patients, with no meals missed by any patient in either group. There were no reported severe adverse events. Pedometer readings revealed no significant difference in the intensity or duration of physical activity between the two groups.

\section{DISCUSSION}

This study compared the effects of two isocaloric diets with different carbohydrate and protein compositions
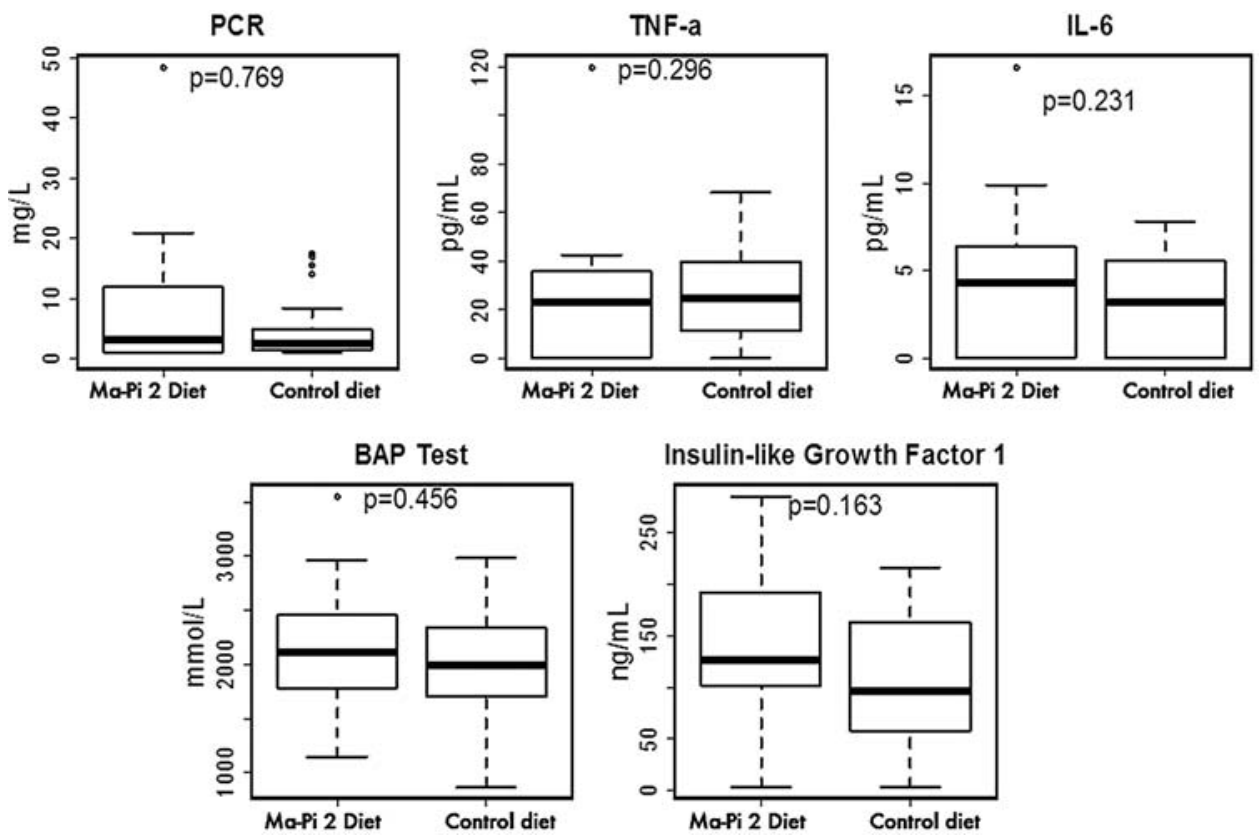

Figure 2 Baseline characteristics of the participants according to study group. The absolute values at baseline (T0) for TNF- $\alpha$ and IL-6, CRP, BAP test, and IGF-1 was graphically represented by means of box-plots. Quantitative variables were summarized using percentiles (median and IQR). $p$ Value by Wilcoxon rank-sum test are referred to differences between the two treatment groups. CRP, C reactive protein; TNF- $\alpha$, tumor necrosis factor- $\alpha$; IL-6, interleukin-6; BAP test, biological antioxidant potential of plasma test; IGF-1, insulin-like growth factor-1. 
Table 2 Change in TNF-a, IL-6, CRP, BAP test, IGF-1, from baseline to T21 in the Ma-Pi 2 group versus control group

\begin{tabular}{|c|c|c|c|}
\hline $\begin{array}{l}\text { Median }\left(1^{\circ}-3^{\circ} \text { quartile) }\right. \\
{[95 \% \mathrm{CI}]}\end{array}$ & Ma-Pi $2(n=25)$ & Control $(n=26)$ & p Value \\
\hline TNF- $\alpha(p g / m L)^{*}$ & $\begin{array}{l}-18(-33 \text { to } 0) \\
{[-28.1 \text { to }-8.1]}\end{array}$ & $\begin{array}{l}-20(-40 \text { to }-5) \\
{[-27.9 \text { to }-4.9]}\end{array}$ & 0.623 \\
\hline IL-6 (pg/mL)* & $\begin{array}{l}0.4(-1.5 \text { to } 2.9) \\
{[-1 \text { to } 1.8]}\end{array}$ & $\begin{array}{l}0.3(-3 \text { to } 3) \\
{[-1.5 \text { to } 2.2]}\end{array}$ & 0.888 \\
\hline CRP (\%)† & $\begin{array}{l}-33.1(-68.8 \text { to } 0) \\
{[-54.9 \text { to }-11.4]}\end{array}$ & $\begin{array}{l}-23.1(-65.5 \text { to } 2) \\
{[-45 \text { to }-1.2]}\end{array}$ & 0.211 \\
\hline BAP test $(\%) \dagger$ & $\begin{array}{l}26.9(9.9 \text { to } 63.8) \\
{[9.1 \text { to } 44.7]}\end{array}$ & $\begin{array}{l}17.9(-2.1 \text { to } 61.3) \\
{[-3.3 \text { to } 39.1]}\end{array}$ & 0.523 \\
\hline IGF-1 (\%)† & $\begin{array}{l}-12.6(-38.3 \text { to }-2) \\
{[-24 \text { to }-1.1]}\end{array}$ & $\begin{array}{l}22.7(0.5 \text { to } 114.6) \\
{[-14.1 \text { to } 59.5]}\end{array}$ & $<0.001$ \\
\hline
\end{tabular}

on anthropometric, biochemical, and inflammatory markers over a 21-day intervention period in patients with T2D. There is an ample body of evidence suggesting that dietary components may modulate inflammatory and oxidative process, ${ }^{18}$ thereby potentially influencing diabetes risk. Epidemiological and clinical evidence concerning diet and inflammation have established that diets high in refined starches, sugar, saturated and trans-fatty acids, poor in natural antioxidants and fiber from fruits, vegetable, and whole grains cause excessive production of proinflammatory cytokines. ${ }^{17}$ T2D appears to be associated with a state of oxidative stress, and an increased intake of antioxidants either from diet or supplements has been shown to have a beneficial influence on markers of T2D and cardiovascular outcomes. ${ }^{29}$

The macrobiotic Ma-Pi 2 diet is rich in complex carbohydrates, whole grains (brown rice, millet, barley), cooked and raw seasonal vegetables (onion, carrot, parsley, chicory, radish, and savoy cabbage), legumes (chickpeas, adzuki beans, lentils, black beans), seaweed, soy sauces, sesame seed, unrefined sea salt, with no added fat or protein from animal sources (including milk and dairy products), and no added sugars. These dietary components were chosen as they have characteristics that may help to achieve optimal glucose control, lower insulin requirement, prolong the time of glucose absorption, increase insulin sensitivity, improve body weight control, and reduce inflammation and oxidative stress.

The higher dietary fiber content in the Ma-Pi 2 diet compared with the control diet probably contributed to the majority of the weight loss, and improved insulin resistance and glucose response in this group. Observational studies suggest that high intakes of fiber and fiber-rich wholegrain foods are independently associated with a reduced risk of obesity. ${ }^{30}$ The Massachusetts Hispanic Elders Study reported that a

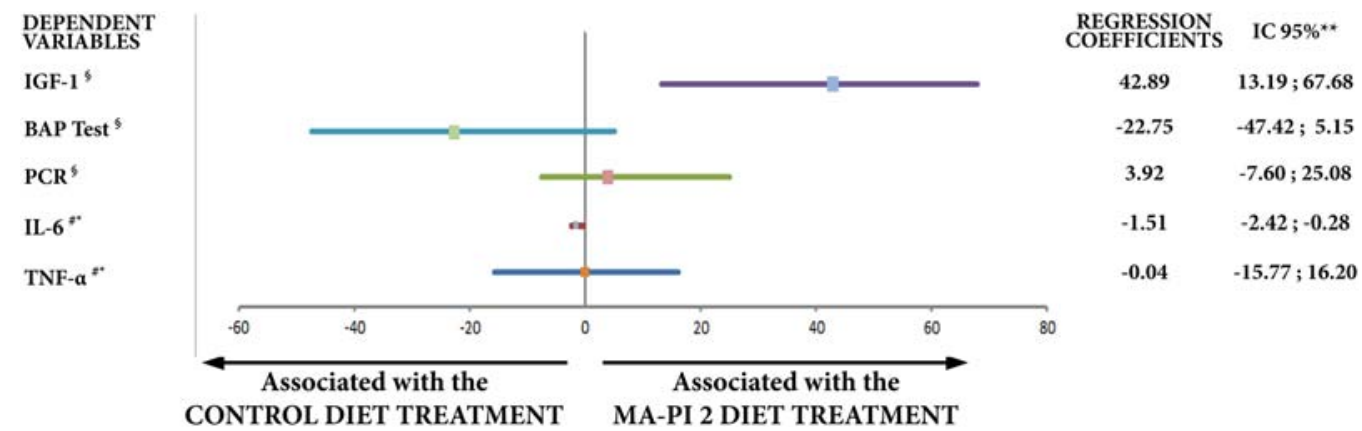

Figure 3 Effect of Ma-Pi diet versus control diet on change in TNF- $\alpha$, IL-6, CRP, BAP test, and IGF-1. Results of multiple quantile regression analysis evaluating the effect of Ma-Pi 2 diet on the percentage or absolute changes (T0-T21) in biochemical measures using a multiple quantile regression model (adjusted for age, gender, physical activity, and BMI at baseline). Variables are expressed as: "absolute difference or ${ }^{\S}$ percent change. ${ }^{*}$ Model adjusted for the variable measured at baseline. ${ }^{\star *}$ Coefficients are statistically significant when the $95 \% \mathrm{Cls}$ do not include zero. CRP, C reactive protein; TNF- $\alpha$, tumor necrosis factor- $\alpha$; IL-6, interleukin-6; BAP test, biological antioxidant potential of plasma test; IGF-1, insulin-like growth factor-1; BMI, body mass index. 
greater frequency of fruit and vegetable intake was associated with lower CRP levels. ${ }^{31}$ Ajani et $a t^{22}$ studied National Health and Nutrition Examination Survey 1999-2000 data and observed that dietary fiber intake was inversely associated with serum CRP levels. In nondiabetic participants from the Insulin Resistance Atherosclerotic Study, Festa $e t a l^{2}$ found significant associations for both insulin resistance and fasting glucose with CRP levels. Whole grains have been recommended over refined grains for the prevention of T2D; a systematic review and meta-analysis of cohort studies found that high whole grain intake was associated with a reduction in the risk of developing T2D. ${ }^{32}$ Vegetable protein intake was the only source of protein in the Ma-Pi 2 diet and may have contributed to the reduction in CRP concentration and the major reduction in IGF-1 levels. Jenkins et al postulated the anti-inflammatory effects of a dietary portfolio treatment in hyperlipidemic adults, wherein a combination of soy proteins, viscous fiber, plant sterols, and almonds lowered CRP levels, similar to the statin-treated group. ${ }^{33}$ Green tea, the main source of liquids in the Ma-Pi 2 diet, was found to be beneficial in reducing fasting glucose and $\mathrm{HbAlc}$ in a meta-analysis of 17 randomized controlled trials. ${ }^{34}$ Green tea contains a group of antioxidant flavonoids. The legumes used in the Ma-Pi 2 diet are particularly rich in phenolic compounds. There is growing evidence that plant-derived polyphenol intake attenuates fasting hyperglycemia and postprandial glycemic response, and improves acute insulin secretion and insulin sensitivity. Dietary polyphenols have gained considerable attention over the past decade because of their potential to modulate the production and activity of inflammatory molecules in humans, decrease plasma levels of TNF- $\alpha$ and IL- 6 , suppress CRP levels, and improve endothelial function in patients with T2D. ${ }^{35}$

Our findings are limited by the relatively small sample size and short intervention period. However, the current results have an important translational value. To our knowledge, this is the first controlled interventional study to evaluate the effects of the macrobiotic Ma-Pi 2 diet on inflammatory status, although these post hoc analysis findings are suitable only for hypothesisgenerating activities. Further study is required in a larger patient population to confirm these positive short-term results and to elucidate whether these initial improvements in inflammatory markers are maintained over the longer term.

In summary, this post hoc analysis found that both the Ma-Pi 2 macrobiotic diet and a standard recommended diet for T2D were found to produce significant reductions in CRP and TNF- $\alpha$ plasma concentration. Of note, after 21 days, participants in the Ma-Pi 2 diet group improved significantly their BAP levels and they had significantly reduced IGF-1 levels compared with the control diet group. We propose that this effect is due to the higher fiber and antioxidant content of Ma-Pi 2. The Ma-Pi 2 diet provides a safe strategy to potentially improve insulin resistance, and to reduce inflammation and the associated cardiovascular risk.

Author affiliations

${ }^{1}$ Area of Endocrinology and Diabetes, University Campus Bio-Medico of

Rome, Rome, Italy

${ }^{2}$ Laboratory Medicine, University Campus Bio-Medico of Rome, Rome, Italy

${ }^{3}$ Center of Integrated Research, University Campus Bio-Medico of Rome,

Rome, Italy

${ }^{4}$ Unit of Dietology and Diabetology, Sandro Pertini Hospital, Rome, Italy

${ }^{5}$ Center of Epidemiology, Biostatistics and Medical Information Technology,

Polytechnic Marche University, Ancona, Italy

${ }^{6}$ In Unam Sapientiam, La Sapienza University, Rome, Italy

${ }^{7}$ International Study Center for Environment, Agriculture, Food, Health and

Economics, Tolentino, Italy

${ }^{8}$ Centre of Diabetes, St. Bartholomew's and The London School of Medicine, Queen Mary University of London, London, UK

Contributors AS conducted the study, recruited participants, collected data and wrote the manuscript. RDT recruited participants, conducted the study and collected data. ER contributed to patients follow-up and reviewed the manuscript. YMK contributed to study design and to the writing of the manuscript. SA and LD analyzed blood samples and reviewed the manuscript. MA and LF recruited participants, contributed to patients follow-up and reviewed the manuscript. SF analyzed data and contributed to the writing of the manuscript. VF and FC collected data and contributed to the writing of the manuscript. RG analyzed data and contributed to the writing and reviewed the manuscript. SM and FF contributed to the study design, to the discussion and reviewed the manuscript. MP conceived and contributed to the study design, and reviewed the manuscript. PP conceived and designed the study, contributed to the discussion and reviewed and edited the manuscript. PP is the guarantor of this work and, as such, had full access to all the data in the study and takes responsibility for the integrity of the data and the accuracy of the data analysis.

Funding This study was supported by 'Un Punto Macrobiotico Association', a charity/not-for-profit organization that provided the food required for the trial and supported the cost of blood analyses. Un Punto Macrobiotico Association has contributed to the costs of the English editing of the text.

Competing interests None.

Patient consent Obtained.

Ethics approval Ethics Committee of the University Campus Bio-Medico of Rome (11/13 PAR ComEt CBM).

Provenance and peer review Not commissioned; externally peer reviewed.

Data sharing statement No additional data are available.

Prior presentation Some of these findings were presented at the 74nd Scientific Sessions of the American Diabetes Association, San Francisco, CA, 13-17 June 2014 (Soare A, Khazrai YM, Angeletti S et al. The effect of macrobiotic (Ma-Pi 2) diet on systemic inflammation in patients with type 2 diabetes. [abstract]. Diabetes 2014;63[Suppl. 1A]:170-212).

Open Access This is an Open Access article distributed in accordance with the Creative Commons Attribution Non Commercial (CC BY-NC 4.0) license, which permits others to distribute, remix, adapt, build upon this work noncommercially, and license their derivative works on different terms, provided the original work is properly cited and the use is non-commercial. See: http:// creativecommons.org/licenses/by-nc/4.0/

\section{REFERENCES}

1. Wild S, Roglic G, Green A, et al. Global prevalence of diabetes: estimates for the year 2000 and projections for 2030. Diabetes Care 2004;27:1047-53

2. Festa A, D'Agostino R Jr, Howard G, et al. Chronic subclinical inflammation as part of the insulin resistance syndrome: the Insulin Resistance Atherosclerosis Study (IRAS). Circulation 2000;102:42-7. 
3. Herder C, Schneitler S, Rathmann W, et al. Low-grade inflammation, obesity, and insulin resistance in adolescents. J Clin Endocrinol Metab 2007;92:4569-74.

4. Shea S, Aymong E, Zybert $\mathrm{P}$, et al. Obesity, fasting plasma insulin, and $\mathrm{C}$-reactive protein levels in healthy children. Obes Res 2003;11:95-103.

5. Xu H, Barnes GT, Yang Q, et al. Chronic inflammation in fat plays a crucial role in the development of obesity-related insulin resistance. J Clin Invest 2003;112:1821-30.

6. Kern PA, Ranganathan S, Li C, et al. Adipose tissue tumor necrosis factor and interleukin- 6 expression in human obesity and insulin resistance. Am J Physiol Renal Physiol 2001;280:E745-51.

7. van Hall G, Steensberg A, Sacchetti M, et al. Interleukin-6 stimulates lipolysis and fat oxidation in humans. J Clin Endocrinol Metab 2003;88:3005-10.

8. Pradhan AD, Manson JE, Rifai N, et al. C-reactive protein, interleukin 6 , and risk of developing type 2 diabetes mellitus. JAMA 2001;286:327-34.

9. Frei B, Higdon JV. Obesity and oxidative stress: a direct link to CVD? Arterioscler Thromb Vasc Biol 2003;23:365-7.

10. Kelishadi R, Hashemi M, Mohammadifard N, et al. Association of changes in oxidative and proinflammatory states with changes in vascular function after a lifestyle modification trial among obese children. Clin Chem 2008;54:147-53.

11. Kim MK, Cho SW, Park YK. Long-term vegetarians have low oxidative stress, body fat, and cholesterol levels. Nutr Res Pract 2012;6:155-61.

12. Benzie IF, Strain JJ. The ferric reducing ability of plasma (FRAP) as a measure of "antioxidant power": the FRAP assay. Anal Biochem 1996;239:70-6.

13. Pinto A, Toselli L, Gavarini G, et al. Dieta, infiammazione e malattie metaboliche. L'Endocrinologo 2014;15:16-21.

14. Gregor MF, Hotamisligil GS. Inflammatory mechanisms in obesity. Annu Rev Immunol 2011;29:415-45.

15. Salas-Salvadó J, Martinez-González MA, Bulló M, et al. The role of diet in the prevention of type 2 diabetes. Nutr Metab Cardiovasc Dis 2011;21:B32-48.

16. Hu FB. Dietary pattern analysis: a new direction in nutritional epidemiology. Curr Opin Lipidol 2002;13:3-9.

17. Hoffmann K, Schulze MB, Schienkiewitz A, et al. Application of a new statistical method to derive dietary patterns in nutritional epidemiology. Am J Epidemiol 2004;159:935-44.

18. Barbaresko J, Koch M, Schulze MB, et al. Dietary pattern analysis and biomarkers of low-grade inflammation: a systematic literature review. Nutr Rev 2013;71:511-27.

19. Giugliano D, Ceriello A, Esposito K. The effects of diet on inflammation: emphasis on the metabolic syndrome. J Am Coll Cardiol 2006;48:677-85.

20. Qi L, Meigs JB, Liu S, et al. Dietary fibers and glycemic load obesity, and plasma adiponectin levels in women with type 2 diabetes. Diabetes Care 2006;29:1501-5.
21. Ma Y, Griffith JA, Chasan-Taber L, et al. Association between dietary fiber and serum C-reactive protein. Am J Clin Nutr 2006;83:760-6.

22. Ajani UA, Ford ES, Mokdad AL. Dietary fiber and $\mathrm{C}$ reactive protein findings from National Health Nutrition Examination Survey data. J Nutr 2004;134:1181-5

23. Soare A, Khazrai YM, Del Toro R, et al; for the MADIAB Group. The effect of the macrobiotic Ma-Pi 2 diet vs. the recommended diet in the management of type 2 diabetes: the randomized controlled MADIAB trial. Nutr Metab 2014;11:39.

24. Associazione Medici Diabetologici, Società Italiana di Diabetologia (Ed). Standard italiani per la cura del diabete mellito. Torino: Edizione per la Medicina Generale Infomedica, 2011.

25. Turner L, Shamseer L, Altman DG, et al. Consolidated standards of reporting trials (CONSORT) and the completeness of reporting of randomised controlled trials (RCTs) published. Cochrane Database Syst Rev 2012;11:MR000030.

26. Gnagnarella $\mathrm{P}$, Parpinel M, Salvini S, et al. The update of the Italian Food Composition Database. J Food Composition Anal 2004;17:509-22.

27. Porrata-Maury $\mathrm{C}$, Hernández-Triana $\mathrm{M}$, Ruiz-Alvarez $\mathrm{V}$, et al. Ma-Pi 2 macrobiotic diet and type 2 diabetes mellitus: pooled analysis of short-term intervention studies. Diabetes Metab Res Rev 2014;30:55-66.

28. Matthews DR, Hosker JP, Rudenski AS, et al. Homeostasis model assessment: insulin resistance and beta-cell function from fasting plasma glucose and insulin concentrations in man. Diabetologia 1985;28:412-19.

29. Bartlett HE, Eperjesi F. Nutritional supplementation for type 2 diabetes: a systematic review. Ophthalmic Physiol Opt 2008;28:503-23.

30. Priebe MG, van Binsbergen JJ, de Vos R, et al. Whole grain foods for the prevention of type 2 diabetes mellitus. Cochrane Database Syst Rev 2008;CD006061. DOI: 10.1002/14651858.CD006061.pub2

31. Gao X, Bermudez OI, Tucker KL. Plasma C-reactive protein and homocysteine concentrations are related to frequent fruit and vegetable intake in Hispanic and non-Hispanic white elders. J Nutr 2004;134:913-18.

32. Aune $\mathrm{D}$, Norat $\mathrm{T}$, Romundstad $\mathrm{P}$, et al. Whole grain and refined grain consumption and the risk of type 2 diabetes: a systematic review and dose-response meta-analysis of cohort studies. Eur J Epidemiol 2013;28:845-58.

33. Jenkins DJ, Kendall CW, Faulkner DA, et al. Assessment of the longer-term effects of a dietary portfolio of cholesterol-lowering foods in hypercholesterolemia. Am J Clin Nutr 2006;83:582-91.

34. Liu K, Zhou R, Wang B, et al. Effect of green tea on glucose control and insulin sensitivity: a meta-analysis of 17 randomized controlled trials. Am J Clin Nutr 2013;98:340-8.

35. Hanhineva K, Törrönen R, Bondia-Pons I, et al. Impact of dietary polyphenols on carbohydrate metabolism. Int $\mathrm{J} \mathrm{Mol} \mathrm{Sci}$ 2010;11:1365-402. 